\title{
Convex and Starshaped Sets in Manifolds Without Conjugate Points
}

\author{
Sameh Shenawy* \\ (Communicated by Yusuf Yaylı)
}

\begin{abstract}
Let $\mathcal{W}^{n}$ be the class of $C^{\infty}$ complete simply connected $n$-dimensional manifolds without conjugate points. The hyperbolic space as well as Euclidean space are good examples of such manifolds. Let $W \in \mathcal{W}^{n}$ and let $A$ be a subset of $W$. This article aims at characterization and building convex and starshaped sets in this class from inside. For example, it is proven that, for a compact starshaped set, the convex kernel is the intersection of stars of extreme points only. Also, if a closed unbounded convex set $A$ does not contain a totally geodesic hypersurface and its boundary has no geodesic ray, then $A$ is the convex hull of its extreme points. This result is a refinement of the well-known Karein-Millman theorem.
\end{abstract}

Keywords: Manifolds without conjugate points; hyperbolic space; convex sets; stars; starshaped sets; extreme points; kernel.

AMS Subject Classification (2010): Primary: 52A10; 52A30; Secondary: 53B20.

\section{An Introduction}

Let $M$ be a $C^{\infty}$ complete Riemannian manifold. A vector field $J$ along a geodesic $\alpha$ is called a Jacobi vector field if

$$
D_{T}^{2} J+\Re\left(\alpha^{\prime}, J\right) \alpha^{\prime}=0,
$$

where $D$ is the covariant derivative and $\Re$ is the curvature tensor. Two points on a geodesic $\alpha$ are said to be conjugate to each other if there is a non-trivial Jacobi vector field along $\alpha$ that vanishes at both of them. A geodesic $\alpha$ has no conjugate points if every Jacobi field along $\alpha$ vanishes at most once. A $C^{\infty}$ complete Riemannian manifold $M$ is called a manifold without conjugate points if every geodesic of $M$ has no conjugate points. In this case, the exponential map is a covering map at every point of $M$. Moreover, if $M$ is simply connected, then $\exp _{p}$ is a diffeomorphism and $M$ has the property that for every two distinct points $p$ and $q$ in $M$, there is a unique geodesic joining them. Let $\mathcal{W}^{n}$ be the class of $C^{\infty}$ complete simply connected $n$-dimensional Riemannian manifolds without conjugate points. The hyperbolic space $H^{n}$, the $n$-dimensional Euclidean space $E^{n}$ and all manifolds with non-positive curvature are good examples of such manifolds. We refer to $[6,7,8,9,10,11,12,17,18,20,21]$ and references therein for more details and examples of these manifolds.

It is very nice to study the boundary of a closed set $A$ in $W \in \mathcal{W}^{n}$ and get global properties of $A$. For instance, the Krein-Milman theorem $[2,14,15,16,19,22]$ in the $n$-dimensional Euclidean space $E^{n}$ asserts that every compact convex set is the convex hull of its extreme points i.e. given a compact convex set $A \subset E^{n}$, one only needs its extreme points $E(A)$ to recover the set shape.

The aim of this paper is to characterize convex and starshaped sets in manifolds without conjugate points using their extreme points. Sufficient conditions for a set $A$ in $W \in \mathcal{W}^{n}$ to be convex, totally geodesic, and starshaped are considered. A generalization of Krein-Milman theorem to the setting of closed unbounded convex sets is given. It is clear that the convex kernel of a starshaped set $A \subset W, W \in \mathcal{W}^{2}$, is the intersection of stars of all points of $A$. In this work, it is proven that, for a compact starshaped set, the convex kernel is the intersection of stars of extreme points only. Moreover, the original starshaped condition is replaced by a more general condition where the intersection of the stars of certain extreme points is not empty. Thus we get a characterization of starshaped sets in $\mathcal{W}^{2}$. 


\section{Results}

Let $W \in \mathcal{W}^{n}$ and let $A$ be a non-empty subset of $W$. The geodesic segment joining two points $p$ and $q$ is denoted by $[p q]$. If $p$ is removed we write $(p q]$. The geodesic ray with vertex at $p$ and passing through $q$ is denoted by $R(p q)$ while the geodesic passing through $p$ and $q$ is denoted by $G(p q)$. We say that $p$ sees $q$ via $A$ if $[p q] \subset A$. The set of all points of $A$ that $p$ sees via $A$ is called the star of $A$ at $p$ and is denoted by $A_{p} . A$ is a starshaped set if there is a point $p \in A$ that sees every point in $A$ i.e. $A_{p}=A$. The set of all such points $p$ is called the kernel of $A$ and is denoted by $\operatorname{ker} A$. $A$ is convex if $\operatorname{ker} A=A$. A point $p \in A$ is called an extreme point of $A$ if $p$ is not a relative interior point of any segment in $A$. The set of all extreme points of $A$ is called the profile of $A$ and is denoted by $E(A)$. Note that, the definition of extreme points is introduced here to a non-convex set so it is somewhat different form the classical one. The convex hull, $C(A)$, of $A$ is the intersection of all convex subsets of $E^{n}$ that contain $A$. Three concepts of convex sets were introduced to complete Riemannian manifolds in [1]. The three concepts coincide in complete simply connected Riemannian manifolds without conjugate points since geodesics of these manifolds are global minimizers.[3, 4, 5, 13, 21].

We begin with the following important lemmas.

Lemma 2.1. Let $W \in \mathcal{W}^{n}$ and let $A$ be a closed subset of $W$. If $a$ and $b$ are points of $A$ and $[a b] \nsubseteq A$, then there are two points $x, y \in \partial A \cap[a b]$ such that $(x y) \cap A=\varphi$.

Lemma 2.2. Let $W \in \mathcal{W}^{n}$ and let $A$ be a compact subset of $W$. Then $A$ has at least one extreme point.

Proof. Let $p$ be in $W \backslash A$. Define the real-valued continuous function $f$ on $A$ by $f(x)=d(p, x), x \in A$. Since $A$ is compact, $f$ attains its maximum value at a point $y \in A$. Thus $A$ is a subset of the closed $\operatorname{disc} \bar{B}(p, r)$ with centre at $p$ and radius $r=d(p, y)$ defined by

$$
\bar{B}(p, r)=\{x \in W: d(p, x) \leq r\}
$$

The point $y$ is an extreme point of $A$ since any geodesic segment containing $p$ in its relative interior cuts the exterior of $A$.

Theorem 2.1. Let $W \in \mathcal{W}^{2}$ and let $A$ be a compact starshaped subset of $W$. Then

$$
\operatorname{ker} A=\bigcap_{x \in E(A)} A_{x}
$$

Proof. Let $B=\bigcap_{x \in E(A)} A_{x}$. By the definition of the kernel of a starshaped set we have

$$
\operatorname{ker} A=\bigcap_{x \in A} A_{x} \subset \bigcap_{x \in E(A)} A_{x}=B
$$

So, we need only to show that $B \subset A$. Let $x \in B \backslash \operatorname{ker} A$. Then there is a point $y \in A$ such that $[x y] \nsubseteq A$. By Lemma 2.1, we find two points $\bar{x}, \bar{y}$ in $\partial A \cap[x y]$ such that $(\bar{x} \bar{y}) \cap A=\phi$. Let $z \in \operatorname{ker} A$, then $z$ sees $\bar{y}$ via $A$ and hence $R(z \bar{y}) \cap A$ is a closed geodesic segment. Let $q \in \partial A$ such that $R(z \bar{y}) \cap A=[z q]$. Suppose that $q \neq \bar{y}$. Since $x \in E(A), q$ sees $x$ via $A$. Then $z$ sees the geodesic segment $[x q]$ via $A$ and consequently $z$ sees $[x \bar{y}]$ via $A$ which is a contradiction and $q$ is not an extreme point i.e. there is a geodesic segment $[a b] \subset A$ such that $p \in(a b)$. It is clear from the choice of $q$ that $(a b) \nsubseteq R(z \bar{y})$. Since $z \in \operatorname{ker} A, z$ sees $(a b)$ via $A$. Thus we get two points $\bar{a}=[z a] \cap[x y]$ and $\bar{b}=[z b] \cap[x y]$ such that $\bar{y} \in[\bar{a} \bar{b}]$ which contradicts the choice of $\bar{y}$. So, $q=\bar{y}$. $\bar{y}$ is not an extreme point otherwise $\bar{y}$ sees $x$. Therefore, we get a geodesic segment $[r s]$ such that $\bar{y} \in(r s) \subset A$. The geodesic $G(r s)$ separates the points $x$ and $z$ otherwise, as we do above, $z$ sees (rs) via $A$ and we get a point $\bar{r}=[z r] \cap[x y] \in A$ that contradicts the choice of $\bar{y}$. Let $H_{1}$ be the closed half space generated by $G(x \bar{y})$ that does not contain $z$ and let $H_{2}$ be the half space generated by $G(z \bar{y})$ that does not contain $x$. Let $D=A \cap H_{1} \cap H_{2}$. $D$ has a non-empty intersection with the geodesic segment $(r s)$ i.e. $D$ has points close to $\bar{y}$. Since $D$ is compact, $D$ has an extreme point $p \in \partial D$ by Lemma 2.2. The boundary points of $D$ are either boundary points of $A$ or points of $G(x \bar{y})$. Thus $p$ is an extreme point of $A$ i.e. $p$ sees $x$ via $A$. Since $z \in \operatorname{ker} A, z$ sees the geodesic segment $[p x]$ via $A$ and consequently $[x \bar{y}] \subset A$ which is a contradiction and the point $x$ does not exist.

Theorem 2.2. Let $W \in \mathcal{W}^{2}$ and let $A$ be a compact subset of $W$. Suppose that $B=\bigcap_{x \in E(A)} A_{x} \neq \varphi$. Then $\operatorname{ker} A=B$ if and only if for every $x \notin A$, there is a geodesic ray with vertex at $x$ having a non-empty intersection with $A$. 
Proof. Suppose that ker $A \neq B$ i.e. $B \nsubseteq \operatorname{ker} A$. Let $y \in B \backslash \operatorname{ker} A$. Thus there is a point $z \in A$ such that $[y z] \nsubseteq A$. Then by Lemma 2.1, there are two pints $\bar{y}$ and $\bar{z}$ in $\partial A \cap[y z]$ such that $(\overline{y z}) \cap A=\phi$. Let $p \in(\overline{y z})$, then we get a point $\bar{p} \notin A$ such that the geodesic ray $R(p \bar{p})$ has a non-empty intersection with $A$. Rotate the ray $R(p \bar{p})$ to touch $\partial A$ such that $p$ is fixed and the angle between $[p \bar{p}]$ and $[p z]$ decreases. The intersection of the new geodesic ray and $A$ has an extreme point $x$ of $A$. Thus $y$ sees $x$ via $A$ and $[x y]$ cuts the geodesic ray $R(p \bar{p})$ in a point $a$ which is a contradiction otherwise $a \in[y x]$ which is also a contradiction. Thus ker $A=B$.

To prove the second implication, let $p \notin A$ and $q \in \operatorname{ker} A$. Consider the geodesic ray $R(q p)$ passing through $p$. The geodesic ray $R(q p) \backslash[q p)$ has a non-empty intersection with $A$ otherwise $q \notin$ ker $A$.

Corollary 2.1. Let $W \in \mathcal{W}^{2}$ and let $A$ be a compact subset of $W$. Then $A$ is starshaped if and only if $\bigcap_{x \in E(A)} A_{x} \neq \phi$ and for every $x \notin A$, there is a geodesic ray with vertex at $x$ having a non-empty intersection with $A$. Moreover, ker $A=\bigcap_{x \in E(A)} A_{x}$.

Theorem 2.3. Let $W \in \mathcal{W}^{n}$ and let $A$ be a non-empty closed subset of $W$. If $\partial A$ is convex, then $A$ is a convex set. Moreover, if $A$ has a non-empty interior, then $A$ is unbounded, $\partial A$ is totally geodesic and $A^{c}$ is also convex.

Proof. Suppose that $A$ is not convex i.e. we get two points $p$ and $q$ in $A$ such that $(p q)$ is not contained in $A$. Since $A$ is closed, we find two points $r, s$ in $\partial A$ such that $(r s) \cap A=\phi$ which is a contradiction and so $A$ is convex.

To show that $A$ is unbounded, let $p \in \operatorname{int}(A)$. Suppose that $A$ is bounded and so we find a real number $\varepsilon$ such that $A$ is contained in the closed ball $\bar{B}(p, \varepsilon)$ of radius $\varepsilon$ and center at $p$. Let $[a b]$ be any chord of $\bar{B}(p, \varepsilon)$ that runs through $p$. Since $A$ and $[a b]$ are both closed and convex sets, we find $a^{\prime}$ and $b^{\prime}$ in $\partial A$ such that $A \cap[a b]=\left[a^{\prime} b^{\prime}\right]$ which is a contradiction since $\left[a^{\prime} b^{\prime}\right]$ cuts the interior of $A$. Therefore $A$ is unbounded.

Assume that $\partial A$ is not totally geodesic i.e. there are two points $a$ and $b$ in $\partial A$ such that the line $G(a b)$ passing through $a$ and $b$ is not contained in $\partial A$. Since $\partial A$ and $G(a b)$ are closed convex sets, there are $a^{\prime}$ and $b^{\prime}$ in $\partial A$ such that

$$
[a b] \subset \partial A \cap G(a b)=\left[a^{\prime} b^{\prime}\right]
$$

Let $p \in G(a b) \backslash\left[a^{\prime} b^{\prime}\right]$ (i.e. $p \in \operatorname{int}(A) \cap G(a b)$ or $p \in A^{c} \cap G(a b)$ ) and assume that $p \in R\left(b^{\prime} a^{\prime}\right)$. If $p \in \operatorname{int}(A) \cap$ $G(a b)$, then the geodesic convex cone $C(b, \bar{B}(p, \varepsilon))$ with vertex $b$ and base $\bar{B}(p, \varepsilon)$ for a sufficiently small $\varepsilon$ shows that $a$ is an interior point which is a contradiction see Figure 1.

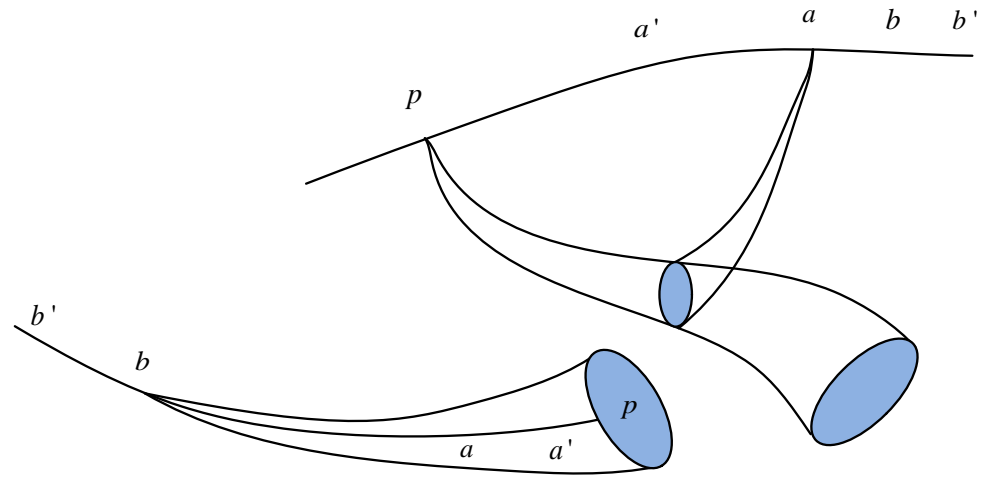

Figure 1. Two cases for the point $p$

Now we take $p \in A^{c} \cap G(a b)$. Let $q$ be a point of $\operatorname{int}(A)$. The sets $[p q]$ and $A$ are closed convex sets and so there is a point $q^{\prime} \in \partial A$ such that $[p q] \cap A=\left[q^{\prime} q\right]$. This implies that the intersection $B=\partial A \cap C(p, \bar{B}(q, \epsilon))$, for a small $\epsilon$, is a non-empty closed convex set since $\partial A$ is convex. Therefore, $B$ is a convex cross section of $C(p, \bar{B}(q, \epsilon))$ that determines a hypersurface $H$ whose intersection with $C(p, \bar{B}(q, \epsilon))$ is $B$. At least one of the points $a$ and $b$ (say $a$ ) does not lie in $H$ otherwise the line $G(a b)$ lies in $H$ which contradicts the fact that $p$ is the vertex of the convex cone $C(p, \bar{B}(q, \epsilon))$. Now, the convex cone $C(a, B)$ has dimension $n$ i.e. $C(a, B)$ has interior points which is a contradiction since both $a$ and $B$ are in $\partial A$ see Figure 1 . This contradiction completes the proof.

Corollary 2.2. Let $W \in \mathcal{W}^{n}$ and let $A$ be a non-empty open subset of $W$ and $\operatorname{int}(\bar{A})=A$. If $\partial A$ is convex, then $A$ is unbounded convex set and $\partial A$ is totally geodesic. 
Proof. It is clear that $\bar{A}$ satisfies the hypothesis of Theorem 2.3. Therefore $\partial \bar{A}=\partial A$ is affine and $\bar{A}$ is convex and unbounded. Note that if $A$ is bounded, then $\bar{A}$ is also bounded and equivalently, $\bar{A}$ is unbounded implies that $A$ is unbounded. Since the interior of a closed convex set is also convex, the convexity of $\bar{A}$ implies that $A$ is convex.

Theorem 2.4. Let $W \in \mathcal{W}^{n}$ and let $A$ be a non-empty closed subset of $W$. If $(p q) \subset \operatorname{int}(A)$ for every pair of boundary points $p, q$ of $A$, then $A$ is strictly convex.

Proof. It is enough to prove that $A$ is convex since the strict convexity of $A$ is direct. Now, we assume that $A$ is not convex i.e. there are $p, q$ in $A$ such that $[p q]$ is not contained in $A$. Since $A$ is closed, there are $p^{\prime}, q^{\prime}$ in $\partial A$ such that $\left(p^{\prime} q^{\prime}\right) \cap A=\phi$ which is a contradiction and $A$ is convex.

Corollary 2.3. Let $W \in \mathcal{W}^{n}$ and let $A$ be a non-empty closed subset of $W . A$ is convex if and only if $(p q) \subset \partial A$ or $(p q) \subset \operatorname{int}(A)$ for each pair of boundary points $p, q$.

Since the interior of a closed convex set is again convex, this result is still true for open sets such that int $(\bar{A})=A$. The following example shows that the closeness is important. Let $A$ be a subset of $E^{2} \in \mathcal{W}^{2}$ defined by $A=\{(x, y): 0 \prec x \prec 1,0 \prec y \prec 1\} \cup\{(0,0),(1,1),(1,0),(0,1)\}$. $A$ is neither closed nor open and $(p q) \subset \partial A$ or $(p q) \subset \operatorname{int}(A)$ for each pair of boundary points $p, q$ but $A$ is not convex.

Proposition 2.1. Let $W \in \mathcal{W}^{n}$ and let $A$ be a non-empty closed subset of $W$. If there is a point $p \in A$ that sees $\partial A$ via $A$, then $A$ is starshaped.

Proof. We claim that $p \in \operatorname{ker} A$. Suppose that $p$ is not in $\operatorname{ker} A$ i.e. there is a point $q \in A$ such that $[p q]$ is not contained in $A$. Since $A$ is closed, there are two points $p^{\prime}$ and $q^{\prime}$ in $\partial A \cap[p q]$ such that $\left(p^{\prime} q^{\prime}\right) \cap A=\phi$. Thus $p$ does not see neither $p^{\prime}$ nor $q^{\prime}$. This contradicts the fact that $p$ sees $\partial A$ via $A$ and the proof is complete.

It is clear that the converse of this result is also true. Thus we can say that this proposition is a characterization of the kernel of the closed starshaped sets. This means that the kernel of a closed starshaped set $A$ is only the points of $A$ that see $\partial A$. The following corollary is direct.

Corollary 2.4. Let $W \in \mathcal{W}^{n}$ and let $A$ be a non-empty closed convex subset of $W$. If $\partial A$ is starshaped, then $\operatorname{ker}(\partial A) \subset$ ker $A$.

In the light of the above results, one can test the convexity and starshapedness of a closed set $A$ using its boundary points. In the next part a minimal subset of these boundary points will build $A$ up from inside.

Theorem 2.5. Let $W \in \mathcal{W}^{n}$ and let $A$ be a non-empty closed convex subset of $W$. If $A$ has no hyperplane, then $A=C(\partial A)$.

Proof. Since $A$ is convex, $A$ is connected. We will prove that $C(\partial A)$ is open and closed in the relative topology on $A$ and hence $A=C(\partial A)$.

First, we prove that $C(\partial A)$ is open in $A$. Let $p \in C(\partial A) \subset A$. We have the following cases:

1. $p \in C(\partial A) \cap$ int $(A)$ : Let $B_{\delta}=B(p, \delta) \cap A$. In this case there exists a real number $\delta$ such that $B(p, \delta) \subset A$ and so $B_{\delta}=B(p, \delta)$. Suppose that $p$ is not an interior point of $C(\partial A)$ i.e. for any $\delta, B_{\delta}$ is not contained in $C(\partial A)$ and so $p$ is a boundary point of $C(\partial A)$. Therefore, there is a supporting hyperplane $H_{1}$ of $\overline{C(\partial A)}$ (the closure of $C(\partial A)$ is a closed convex subset of $A$ ) at $p$ and $\overline{C(\partial A)}$ is contained in a closed halfspace with boundary $H_{1}$. Let $x$ be any point of $B(p, \delta)$ that lies on the other side of $H_{1}$ and let $H_{2}$ be a parallel hyperplane to $H_{1}$ at $x$. Since $A$ does not contain a hyperplane, we find a point $y \in H_{2} \backslash A$. The line segment $[x y]$ cuts $\partial A$ at a point $z \in H_{2}$ which contradicts the fact that $H_{1}$ supports $\overline{C(\partial A)}$. This contradiction implies that $p$ is an interior point of $C(\partial A)$ in the relative topology of $A$.

2. $p \in C(\partial A) \cap \partial A$ : in this case, $B(p, \delta)$ has a non-empty intersection with $A$ for any real number $\delta$. Let $B_{\delta}=B(p, \delta) \cap A$. Suppose that $p$ is not an interior point of $C(\partial A)$. Then, for any $\delta$, the set $B_{\delta}$ has a point $x$ which is not in $C(\partial A)$. But $\overline{C(\partial A)}$ is closed convex set and $x \notin \overline{C(\partial A)}$, and so we get a hyperplane $H$ passing through $x$ that separates $x$ and $\overline{C(\partial A)}$. Since $A$ does not have a hyperplane, there is a point $y$ in $H \backslash A$ where $[x y]$ cuts $\partial A$. Thus $H$ cuts $\partial A$ and so $H$ cuts $C(\partial A)$ which is a contradiction and so $p$ is an interior point of $C(\partial A)$ in the relative topology on $A$. 
This discussion above implies that $C(\partial A)$ is an open set in $A$. Now, we want to prove that $C(\partial A)$ is closed in $A$. Let $p$ be a boundary point of $C(\partial A)$. If $p \in \partial A$, then $p \in C(\partial A)$. Let $p \in i n t A$, then there is a small positive real number $\delta$ such that $B(p, \delta) \subset A$. Since $p$ is a boundary point of $C(\partial A), B(p, \delta) \neq B(p, \delta) \cap C(\partial A) \neq \phi$. Therefore, we find a point $x$ in $B(p, \delta)$ which is not in $C(\partial A)$. Since $\overline{C(\partial A)}$ is a closed convex set, we get a hyperplane $H$ passing through $x$ and does not intersect $C(\partial A)$. But $A$ does not have a hyperplane and so $H$ cuts $\partial A$ which is a contradiction and $p \in C(\partial A)$ i.e. $C(\partial A)$ is closed in the relative topology on $A$ and the proof is complete.

In general, sets need not have extreme points. The following proposition gives a sufficient condition for the existence of extreme points.

Proposition 2.2. Let $W \in \mathcal{W}^{n}$ and let $A$ be a non-empty closed convex subset of $W$. A contains at least one extreme point if and only if $A$ has no geodesic.

Proof. Let us assume that $A$ has a geodesic $l$. Suppose that $A$ has an extreme point $p$. It is clear that $p \notin l$. Let $B$ be the closed convex hull of $p$ and $l$. $B$ is a subset of $A$ since $A$ is a closed convex set containing both $p$ and $l$. It is clear that $B$ contains a line passing through $p$ and parallel to $l$ i.e. either $p$ is not an extreme point or the line $l$ does not exist.

Lemma 2.3. Let $W \in \mathcal{W}^{n}$ and let $A$ be a non-empty closed convex subset of $W$. If $H$ is a supporting totally geodesic hypersurface of $A$, then $E(H \cap A) \subset E(A)$

Proof. Let $p$ be an extreme point of $H \cap A$. Suppose that $p \notin E(A)$ i.e. there are $x, y$ in $\partial A$ such that $p \in(x y)$. The hypersurface $H$ supports $A$ at $p$ and so $[x y] \subset H$. This implies that $p \in[x y] \subset H \cap A$ which contradicts the fact that $p$ is an extreme point of $H \cap A$. This contradiction completes the proof.

The minimal subset of a compact convex set $A$ which generates $A$ is is its extreme points. Our next main theorem shows that this property is more general.

Theorem 2.6. Let $W \in \mathcal{W}^{n}$ and let $A$ be a non-empty closed convex subset of $W$. If $A$ has no hyperplane and its boundary has no ray, then $A=C(E(A))$.

Proof. To prove that $A=C(E(A))$, it suffices to prove that $\partial A \subset C(E(A))$ and by Theorem 2.5, we get that $A=C(\partial A) \subset C(E(A)) \subset A$ and hence $A=C(E(A))$. Let $p \in \partial A$. If $p$ is an extreme point, then $p \in E(A) \subset$ $C(E(A))$. Now suppose that $p$ is not an extreme point i.e. there are $x, y$ in $\partial A$ such that $p \in(x y)$. Since $A$ is a closed convex set, there is a supporting totally geodesic hypersurface $H$ of $A$ at $p$. It is clear that the set $H \cap A$ is a non-empty closed convex subset of $\partial A$. Since $\partial A$ has no ray, the set $H \cap A$ is bounded i.e. $H \cap A$ is a compact convex set. Therefore, $H \cap A=C(E(H \cap A))$. But $E(H \cap A) \subset E(A)$ and so $p \in C(E(H \cap A)) \subset C(E(A))$ and the proof is complete.

\section{References}

[1] Alexander, S., Local and global convexity in complete Riemannian manifolds. Pacific Journal of Mathematics 76(1978), no. 2 , 283-289.

[2] Balashov, M. V., An Analog of the Krein-Mil'man Theorem for Strongly Convex Hulls in Hilbert Space. Mathematical Notes 71(2002), no. 1-2, 34-38.

[3] Beltagy, M., Sufficient conditions for convexity in manifolds without focal points. Comment. Math. Univ. Carolinae 34 (1993), $443-449$.

[4] Beltagy, M., Local and global exposed points. Acta Mathematica Scientia 15(1995), no. 3 , 335-341.

[5] Beltagy, M., On starshaped sets. Bull. Malays. Math. Soc., II. Ser. 11(1988), no. 2 , 49-57.

[6] Burns, K., The flat strip theorem fails for surfaces with no conjugate points. Proceedings of the American Mathematical Society 115(1992), no. 1, 199-206.

[7] Eberlein, P., Geodesic flow in certain manifolds without conjugate points. Transactions of the American Mathematical Society 167 (1972), 151-170.

[8] Emmerich, P., Rigidity of complete Riemannian manifolds without conjugate points. Shaker Verlag Gmbh, Germa, 2013.

[9] Green, L. W., Surfaces without conjugate points. Transactions of the American Mathematical Society 76(1954), no. 3 , $529-546$.

[10] Goto, M. S., Manifolds without focal points. Journal of Differential Geometry 13(1978), no. 3 , $341-359$.

[11] Gulliver, R., On the variety of manifolds without conjugate points. Transactions of the American Mathematical Society 210 (1975), $185-201$.

[12] Ivanov, S. and Vitali K. Manifolds without conjugate points and their fundamental groups. Journal of Differential Geometry 96(2014), no. 2 , 223-240.

[13] Jaume, D. A. and Rubén, P., Conjugacy for closed convex sets. Contributions to Algebra and Geometry 46 (2005), no. 1, $131-149$.

[14] Lay, S.R., Convex sets and their applications. Courier Corporation, 2007.

[15] Li, S. and Yicheng G., On the relations of a convex set and its profile. In Integral Geometry and Convexity pp. 199-211. 2006. 
[16] Martinez-Maurica, J. and Garcia, P. C. , A new approach to the Krein-Milman theorem. Pacific Journal of Mathematics 120(1985), no. 2 , 417-422.

[17] Morse, M. and Gustav A. H., Manifolds without conjugate points. Transactions of the American Mathematical Society 51 (1942), $362-386$.

[18] O'Sullivan, J.J., Manifolds without conjugate points. Mathematische Annalen 210(1974), no. 4 , $295-311$.

[19] Oates, D. K., A non-compact Krein-Milman theorem. Pacific Journal of Mathematics 36(1971), no. 3 , 781-785.

[20] Ruggiero, R.O., A note on the divergence of geodesic rays in manifolds without conjugate points. Geometriae Dedicata 134(2008), no. 1 131-138.

[21] Shenawy, S., Tietze-type theorem in 2-dimensional Riemannian manifolds without conjugate points. Balkan Journal of Geometry and Its Applications 16(2011), no. 2 , 133-137.

[22] Valentine, Frederick A., Convex sets. McGraw-Hill, Inc., New York 1964.

\section{Affiliations}

\section{SAMEH SHENAWY}

ADDRESS: Basic Science Department, Modern Academy for Engineering and Technology, Maadi, Egypt.

E-MAIL: drssshenawy@eng.modern-academy.edu.eg, drshenawy@mail.com

ORCID ID: 0000-0003-3548-4239 